\title{
El liderazgo y la comunicación desde la perspectiva de los seguidores de las empresas Cerveceras
}

\author{
Leadership and communication from the perspective of the followers \\ of the beer companies
}

\section{Liderança e comunicação na perspectiva dos seguidores das cervejarias}

\author{
《L Linda Josefina Barboza Osorio \\ lindisima0104@hotmail.com \\ Universidad José Antonio Páez, Venezuela
}

Recibido: 03-03-2018 / Revisado: 09-03-2018 / Aceptado: 19-03-2018 / Publicado: 10-09-2018

\section{RESUMEN}

La investigación tuvo como objetivo central, analizar el liderazgo y la comunicación desde la perspectiva de los seguidores de las empresas "Cervecería Polar de la Quizanda C.A." y "Alimentos Polar C. A, Planta de Limpieza". La metodología del estudio, se desarrolló bajo un enfoque cualitativo, la teoría fundamentada y un diseño sistemático. La muestra estuvo compuesta por (3) unidades de expertos de máxima variación. En consecuencia, los datos cualitativos se recolectaron por medio de la bitácora y la entrevista cualitativa. Posteriormente, se procesaron por medio de la categorización y al obtener la categoría central se vinculó a la teoría para luego redactar el informe final. Como conclusión, se señala que desde la perspectiva de los seguidores la organización posee un liderazgo innovador, creativo, capacitado, dinámico, perseverante, proactivo, con habilidades de flexibilidad y adaptación al contexto; lo que combina aspectos del liderazgo Situacional II y Visionario.

Palabras clave: Liderazgo; comunicación; contexto; perspectiva, seguidores

\section{ABSTRACT}

The main objective of the research was to analyze leadership and communication from the perspective of the followers of the companies "Cerveceria Polar de la Quizanda C.A." and "Alimentos Polar C. A, Cleaning Plant". The study methodology was developed under a qualitative approach, grounded theory and a systematic design. The sample consisted of (3) expert units of maximum variation. Consequently, qualitative data were collected through the blog and qualitative interview. Subsequently, they were processed through categorization and upon obtaining the central category, it was linked to the theory and then the final report was written. In conclusion, it is pointed out that from the perspective of the followers, the organization has innovative, creative, trained, dynamic, persevering, proactive leadership, with flexibility and adaptation skills to the context; which combines aspects of Situational II and Visionary leadership..

Key words: Leadership; communication; context; perspective followers

\section{RESUMO}

O objetivo principal da pesquisa foi analisar a liderança e a comunicação na perspectiva dos seguidores das empresas "Cervecería Polar de la Quizanda C.A." e "Alimentos Polar C. A, Cleaning Plant.". A metodologia do estudo foi desenvolvida sob uma abordagem qualitativa, teoria fundamentada e um desenho sistemático. A amostra foi composta por (3) unidades especialistas de variação máxima. Consequentemente, os dados qualitativos foram coletados por meio do blog e da entrevista qualitativa. Posteriormente, foram processados por meio de categorização e, ao obter a categoria central, foi vinculada à teoria e, em seguida, redigido o relatório final. Concluindo, destaca-se que na perspectiva dos seguidores a organização possui liderança inovadora, criativa, treinada, dinâmica, perseverante, proativa, com flexibilidade e capacidade de adaptação ao contexto; que combina aspectos de Situacional II e liderança Visionária.

Palavras-chave: Liderança; comunicação; contexto; seguidores em perspectiva 


\section{INTRODUCCIÓN}

$\mathrm{E}$ I liderazgo hace referencia a una práctica que ha sido objeto de muchas investigaciones sociales, esta comprende desde el perfil de los líderes, su evolución y adaptación en el tiempo, hasta la identificación de los elementos, circunstancias que lo generan y lo ubican en los grupos sociales. Es innegable que, la sociedad actual está deseosa de ser conducida por líderes que sean agentes de constante cambio. Por otra parte, las organizaciones demandan una nueva generación de directivos, capaces de respetar procesos, inspirar una visión que pueda ser compartida, despertar el ánimo de los seguidores y conducir de manera adecuada la inconformidad, el desasosiego y la inestabilidad del entorno, así como capacitar a otros para actuar.

Puede reconocerse a los líderes como pioneros por naturaleza, se atreven a salir de lo convencional y buscan nuevas formas de hacer las cosas. Esta práctica la realizan mediante dos compromisos: La búsqueda de oportunidades y la toma de decisiones. No obstante, el líder debe enfrentar retos cotidianamente, y de ese modo desarrollar habilidades para dirigir el cambio e influir en su rumbo, dirección y amplitud, unos lo logran, otros no. Por lo tanto, se puede decirse que el liderazgo es un proceso mediante el cual sistemáticamente un individuo ejerce influencia permanente sobre otros en el desarrollo de funciones grupales en un tiempo considerable.

Para ello, esta figura necesita desarrollar con eficacia un proceso de comunicación en la organización, la cual es importante para lograr la eficiencia en el liderazgo y como consecuencia promover el cambio. A ésta, le puede concebir como un elemento esencial e imprescindible para la vida de las organizaciones, el buen funcionamiento, creación y mantenimiento de la cultura organizacional, así como la identidad corporativa.

Basa en que, cuando ocurre la comunicación organizacional se establece un esfuerzo creativo y certero dentro de las relaciones humanas. Este proceso incluye no solo al liderazgo, sino también a sus seguidores. Lo que motivó esta investigación fue dirigirse a estudiar la percepción que tienen los seguidores acerca del liderazgo y la comunicación dentro de las Empresas Polar, C. A. Entonces, para dar respuesta a este fin planteado se propuso determinar el perfil del liderazgo y describir el proceso de comunicación entre estas dos figuras.

\section{Marco teórico}

Sin desestimar que, el entorno mundial ha evolucionado sin precedentes en los últimos años en este Siglo XXI y, el ser humano se percibe desde una perspectiva más amplia dentro diversos campos, en un contexto de avance continuo donde se manifiesta una nueva concepción del Liderazgo. Es curioso y relevante a su vez que, en este mundo globalizado y competitivo, se profundice en la necesidad de potenciar una nueva generación de líderes que resulten innovadores. Lo cual ha llevado a captar la atención de académicos, investigadores y organizaciones. Esto, resulta un tema polémico y que puede ser conceptualizado de diferentes maneras, pero, en lo que existe coincidencia es en 
concebir a los líderes como seres capaces de exhibir ciertos atributos en su personalidad que se manifiestan en sus conductas y los habilitan para conducir a otros.

Por tanto, Pardo (2015) citando a Maxwell refiere lo siguiente: "El liderazgo se desarrolla, no se manifiesta. El verdadero líder "nato" siempre surgirá, pero para permanecer en la cúspide debe desarrollar características propias del liderazgo" (p. 98). En consecuencia, el líder logrará metas mediante sus habilidades, bien sean intra e interpersonales y la manera en que es capaz de motivar a su equipo de trabajo. En síntesis, existe liderazgo cada vez que alguien guía e influye en la conducta de un grupo o individuo.

Es decir que, en el caso de las organizaciones representa la oportunidad de establecer una relación entre el que dirige y los que le siguen con fines productivos y así garantizar el desarrollo y sustentabilidad que beneficiará a todos los que en participen. Por otra parte, cuando el ambiente se torna inestable y cambiante, las organizaciones deben enfrentar retos de desempeño para poder lograr la supervivencia. Por tanto, se hace necesario combinar tres elementos: estrategia, liderazgo y compromiso. La primera define objetivos y rumbo, el segundo la conecta y la transmite a los seguidores y el andar de la empresa dependerá del tercero que las políticas y líderes generen.

Apenas naciendo el siglo XXI, Venezuela muestra un contexto institucional inestable, cambiante y cada vez más regulado, en medio de un decrecimiento económico. Lo cual, obliga a las organizaciones a desarrollar nuevas maneras de adaptarse, sobrevivir y aprovechar las oportunidades. Algunos autores como (Schein, 1992; Wilderom y Van den Berg, 2000) citados por IESA (2016) plantean que existe una relación entre el liderazgo y el desempeño de las empresas.

Dentro de este panorama, y bajo estas condiciones dadas en Venezuela las empresas del sector privado se han visto en la necesidad de implementar grandes cambios, de modo que, puedan salir airosas en medio de esta grave crisis que atraviesa el país. Es decir, tienen la necesidad de un nuevo liderazgo para hacerle frente a la situación, con características muy particulares que involucren poder en la comunicación y las nuevas herramientas tecnológicas, reconocimiento, manejo de las emociones y flexibilidad en el desempeño, para responder al panorama empresarial ya antes descrito.

Es innegable que, el liderazgo en la actualidad no necesariamente tiene que ver con la posición jerárquica que ocupa una persona dentro de una organización. Sino más bien, con un proceso de mutuo reconocimiento y aceptación entre el líder y sus seguidores, donde, el primero asume su papel como tal y conoce a quienes lo siguen, y así los otros le reconocen a él. De otro modo, puede decirse que no existe dicha figura. Viene al caso señalar que, la gente quiere seguir a líderes que sean congruentes, es decir, que gocen de credibilidad.

A través de los años, se ha tratado de determinar de manera consistente las cualidades que deben sobresalir en éstos, tales como: honestidad, visión, ser capaz de inspirar, experimentado, de buen juicio y sobre todo muy hábil en el proceso de comunicar a sus seguidores. Respecto al tema, López (2011) aporta lo siguiente: "Mientras más competencia de liderazgo tiene una persona, con mayor prontitud reconoce el liderazgo -o la falta del mismo- en otros" (p. 1). 
Por ello, al principio las personas dentro de un equipo pueden desenvolverse erráticamente en diversas direcciones, pero al conocerse entre sí, ocurre casi de inmediato que reconozcan a los líderes de mayor potencial y comiencen a seguirlos. Como consecuencia, este tipo de relación entre líderes y seguidores promueve el desarrollo del sentido de pertenencia, un fuerte espíritu de trabajo en equipo y conlleva a percibir sus propios valores como consistentes con los de la organización. Lo cual, se hace necesario para la operatividad cotidiana dentro de toda estructura.

En opinión de Barroso (2011) y referente a lo tratado, puede notarse una carencia en Venezuela:

Nuestro problema fundamental es de ausencia del liderazgo. Se requiere de un liderazgo de presencia de alta credibilidad, para dar la batalla contra una manera de pensar, de sentir y desempeñarse que no nos ha permitido tener calidad en lo que hacemos y gerenciar de distinta manera el diario quehacer...Presencia quiere decir la energía contagiosa de la autoestima, del cultivo de la vida interior, del contacto, de la efectividad en sus acciones, y con la decisión de que las cosas se hagan de manera diferente (p. 345).

En esta cita, se dilucida la necesidad de una figura con alto desempeño, de credibilidad, reflexiva e inspiradora en su proceder, comprometida con lo que hace y con aquellos que dependen de él. $Y$ como complemento, que sea capaz de comunicar, transmitir su pasión, un modelo a seguir por lo seguro y pertinente de sus acciones. Esto significa que, según lo aportado anteriormente por López y Barroso, el viaje hacia la construcción de un nuevo liderazgo exige tanto a los líderes como a sus seguidores poner en tela de juicio algunos de los supuestos más básicos con respecto a cómo deben estos, operar dentro de las organizaciones.

Debido a estas razones, anunciar un cambio no es suficiente, los que dirigen deben dominar nuevas habilidades y aprender a confiar en los individuos, desarrollar un nuevo estilo de comunicación de modo que puedan hacerles comprender a éstos que forman parte de un tejido organizacional que les faculta para la toma de decisiones, les capacita para trabajar como parte de un equipo y espera lo mejor de ellos. Referente al liderazgo, la relación con la comunicación y los desafíos que presenta la actualidad empresarial venezolana, Castejón, citado por Pernía 2013, refiere lo siguiente:

El liderazgo es comunicación, para entender los objetivos corporativos y personales, conformando acuerdos y compromisos tan poderosos que permitan el ejercicio de la autoridad de manera natural... Uno de sus principales retos ha sido operar con las condiciones que el país presenta, lo que obligó a afinar los objetivos corporativos, sincronizando las iniciativas regionales con acción local (p.1).

De lo cual puede entenderse que, el elemento comunicador es inherente al liderazgo para cumplir con el proceso de administrativo, la toma de decisiones y determinar la dirección, pero desde un nuevo y flexible ángulo en la gestión de todo directivo, con características muy particulares, que se articulan a 
la realidad de cada cotidianidad y localidad. En ese orden de ideas, Pernía (2013) describe el escenario entre el liderazgo y sus seguidores del siguiente modo:

Como la polarización social, la lógica de la "lucha de clases", ha entrado en muchas empresas, los gerentes han entendido que necesitan herramientas nuevas y más complejas para garantizar que se sigan cumpliendo los objetivos. Las relaciones con sindicatos más "empoderados" o con colectivos laborales más conscientes de sus derechos, se han hecho más difíciles y tratar de resolver las fuentes de conflicto, incluso antes que la crisis estalle, se ha hecho prioritario. Los conflictos pueden surgir de cualquier cosa o detalle insignificante. Muchos aparecen por causas que no son objetivas, sino sujetas a interpretación y la interpretación del trabajador es prioritaria (p. 1).

Es incuestionable que esta nueva situación, es resultante de una serie de cambios en la estructura jurídica, económica y social que se han originado en el país. Y, por ende, impulsan el desafío de liderizar en tiempos difíciles, de transición, en un contexto lleno de dificultades externas e internas para las empresas, que involucran a los seguidores y que no admiten ser desestimadas, por el contrario, se debe apreciar mucho el "como" los seguidores perciben el liderazgo y la habilidad de quienes les dirigen para comunicar con claridad, efectividad, responsabilidad y ética.

Refiriéndose a la importancia de esta última dentro de la organización Kreitner y Kinicki (2008) exponen lo siguiente:

La comunicación es importante para impartir las directrices respecto a las normas éticas y las actividades de integración de las distintas áreas funcionales de la empresa... Por otra parte, la comunicación eficaz reviste importancia trascendental para el éxito de la gestión y de la organización (p. 400).

Entonces, es demostrable en el orden lógico de ideas antes expuestas que, la comunicación es un elemento fundamental en el desarrollo de un liderazgo efectivo hacia los seguidores, de modo que se desarrolle la habilidad para mantener al grupo de trabajo altamente motivado y comprometido. Así, se promueve en el líder la destreza para reaccionar acertadamente, ser proactivo y adoptar decisiones adecuadas que permitan la adaptación mejor posible a la situación cotidiana.

No cabe duda en lo descrito hasta el momento, la percepción de una realidad que requiere ser abordada y de ese modo, arrojar una nueva perspectiva para que los lideres puedan hacerle frente a esta situación social, económica e histórica. Lo que promueve el análisis de las situaciones cotidianas e interacción de manera exitosa con quienes se comparte la responsabilidad de lograr la estabilidad y el objetivo deseado. Desde el punto de vista de esta investigación se busca analizar la perspectiva que puedan tener los seguidores acerca de quienes los dirigen en cuanto a reconocer el tipo de liderazgo y 
la capacidad de comunicación organizacional en un contexto en crisis. Ya que el resultado de dicho análisis, será una herramienta para el éxito de las empresas y la permanencia en el mercado.

Cabe resaltar que, para este caso fue objeto de estudio dos de las empresas que conforman la organización de las Empresas Polar C.A. Como son la "Cervecería Polar C.A. Sede La Quizanda" y "Alimentos Polar, C. A". (Planta de Mantenimiento), Municipio Valencia del Estado Carabobo, y el sujeto de estudio es el liderazgo de dicha organización. Debe destacarse al grupo de Empresas Polar, como una organización reconocida por el pueblo venezolano como tradicional y de mucha valía en el ramo de la alimentación y bebidas desde hace más de 50 años. Enfatizando, el empeño de seguir siendo pioneros e innovadores a pesar del comportamiento del contexto económico-político y social que ha impactado al aparato productivo del país; razón por la cual, originó esta investigación a fin de conocer este fenómeno emergente y que pueda ser de guía para otras organizaciones.

\section{MÉTODO}

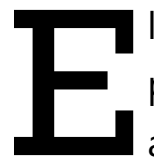

I estudio se desarrolló bajo el enfoque cualitativo, esta perspectiva, consiste en obtener el punto de vista de los participantes, sus emociones, experiencias y significados, así como, otros aspectos subjetivos la cual es definida por Hernández, Fernández y Baptista (2008) de la siguiente manera:

Las investigaciones cualitativas se fundamentan más en un proceso inductivo (explorar y describir, y luego generar perspectivas teóricas). Van de lo particular a lo general. El enfoque cualitativo: Utiliza la recolección de datos sin medición numérica para descubrir o afinar preguntas de la investigación en el proceso de interpretación (p.8).

Es decir, existe mucho interés en los individuos, grupos, colectividades, organizaciones, entre otros. Debido a que, el investigador interpreta las perspectivas y conductas de las personas objeto de estudio, reconociendo las tendencias personales.

En ese orden de ideas, se seleccionó de este enfoque el tipo Teoría Fundamentada, esto indica que se relaciona con una situación y contexto particular.

Por ello, se consideró pertinente el diseño, ya que, se buscó comprender los cambios ocurridos en dos organizaciones del Grupo "Empresas Polar" en el desempeño de su liderazgo, interpretando así la manera en que comunican e interactúan los líderes y seguidores, a fin de definir el rol de la comunicación en la percepción de éstos últimos, en un contexto económico, social y político bastante convulso, como es el caso de Venezuela durante los periodos gubernamentales 2013-2018.

De acuerdo con el caso, fue seleccionado el diseño "sistemático" Lo que indica una serie de pasos que permiten un manejo organizado y procedimental que complementa y da una base formal a la información recolectada. 
Por consiguiente, la muestra, se encontró comprendida por trabajadores, los cuales fueron observados en su contexto interno organizacional, y son quienes aportaron sus percepciones acerca del tema antes mencionado, desde su punto de vista y como personas que lo experimentan día a día. Antes de penetrar en el campo de investigación, se seleccionaron las unidades de muestra. Debido a ello, se escogió un grupo de (3) personas, una del departamento de administración, otro encargado del departamento de saborización y el tercero corresponde a un trabajador del departamento de almacén que opera como montacarguista. Además, un líder de la gerencia y dos seguidores que dieron a conocer la perspectiva que tienen acerca de sus líderes y la comunicación. Tales muestras son válidas y útiles, pues los objetivos del estudio así lo requirieron.

Durante la investigación, la recolección de los datos ocurrió dentro de un ambiente natural y cotidiano donde se desenvolvieron los participantes, que antes se definieron como unidades de análisis. Estás, se reconocieron como fundamental, ya que, estos datos se convirtieron en información.

Considerando el orden, para apreciar las características descritas se implementaron las técnicas recolección de información, que permitieron en el breve tiempo estimado, dar forma y aplicar los instrumento, pero sin obviar adentrarse en profundidad en situaciones sociales, así como, una reflexión permanente. Estar atento a los detalles, sucesos, eventos e interacciones. De ese modo, se pudo proceder a observar el contexto y las interrelaciones humanas, mientras se desarrollaba el encuentro. Esto implicó el añadir a este nuevo ejercicio, los sentidos para percibir al ambiente y sus actores, ya que algunos detalles fueron relevantes.

También, el desarrollo y puesta en práctica de un plan que guio a reunir la información de interés para este estudio, en este sentido, se utilizaron las técnicas de: observación de documentos, observación directa con su respectivo registro e instrumentos. En este caso, se observaron documentos tales como antecedentes de la investigación, artículos de revistas especializadas en liderazgo y teorías que se vincularon a la investigación.

Posterior a ello, se incluyó la entrevista cualitativa en profundidad con un listado de temas, la cual fue íntima, flexible y abierta. Es decir, se utilizó una guía de temas que permitieron la flexibilidad de manejar una variedad de ítems, de manera que se pudo extraer en medio de ese proceso, los datos más adecuados, según fueron fluyendo en la interacción y el ambiente.

Finalmente, se procedió a desarrollar el proceso de análisis fundamental de los datos cualitativos recolectados a través de la entrevista, se procedió a organizar, transcribiendo los datos de las entrevistas, seleccionando lo relevante y que aportara claridad y oportunidad de reflexión, entre otros. Posteriormente, se revisaron los datos por medio de la lectura y la observación a fin de conseguir un panorama general de los materiales y se procedió a la realizar la categorización.

Por consiguiente, y debido a la naturaleza de la teoría fundamentada, el diseño para el análisis de los datos fue sistemático. Todo este procedimiento, permitió la observación y revisión de los segmentos del material analizado y generó comparaciones y significados, eliminó la redundancia y 
desarrolló evidencia producto de la reflexión. Prosiguiendo así, con el tratamiento de los datos recopilados, donde se redujeron, expresaron y describieron de una manera conceptual en una estructura sistemática, significativa e inteligible que van de lo conceptual, esquemas e informe.

Después de todo el procedimiento antes explicado, se puede comparar a la investigación cualitativa como un diseño artesanal, algo elaborado pacientemente, con sabiduría de la práctica continua, de comprensión incluso, donde el investigador imprime las características y costumbres del contexto, las propias y logra un producto único y valioso.

\section{RESULTADOS Y DISCUSIÓN}

Los resultados de este estudio lograron los siguientes hallazgos:

\section{1.- Caracterizar en su contenido y expresión la percepción que tienen los seguidores del liderazgo en las Empresas Polar C.A.}

Desde la percepción de los seguidores el liderazgo es visto con un perfil Creativo, dinámico y proactivo, por una parte y por la otra, es flexible, esto lo hace adaptable a los cambios. Lo que le permite adoptar diversos tipos de liderazgo en diferentes circunstancias. Según la teoría del "Liderazgo Situacional II" propuesta por Ken Blanchard y Paul Hersey en 2011, quienes desarrollaron una estrategia de ajuste y revisión de la teoría originaria de los años 60 , postulan lo siguiente:

Un enfoque eficaz para el manejo y la motivación de las personas por cuanto abre la comunicación y fomenta la alianza entre el líder y los colaboradores que aquél apoya y del cual dependen, El modelo puede resumirse con esta frase: Diferentes estilos para diferentes personas. El liderazgo Situacional II se basa en las suposiciones de que las personas pueden y quieren desarrollarse y de que no hay un estilo de liderazgo óptimo para estimular tal desarrollo. El estilo de liderazgo debe adaptarse a la situación (p.115-116).

Se entiende entonces, como la capacidad que deben poseer quieres dirigen a otros para hacer uso del tipo de liderazgo que amerite la situación que se está viviendo. Todo ello, permite una mayor comunicación con sus colaboradores a fin de que este pueda ayudarles a desarrollarse a cualquiera que sea la demanda del momento. Es así como, Blanchard y Hersey, Dirección, entrenamiento, apoyo y delegación; que comprende: las etapas, con los niveles de desarrollo del individuo, comportamiento de los directivos y estilos de decisiones.

Sumado esto, a la influencia que ejercen en sus seguidores por medio de estar conscientes y ejercitar los valores empresariales, manteniendo el respeto mutuo, diálogo directo y responsable. Esto, hace de ellos un modelo a seguir. Lo que es respaldado por la teoría del "Liderazgo Visionario". Sin 
desestimar que, existe conciencia de algunas debilidades en el mensaje transmitido que pueden debilitar la influencia y la motivación.

Es interesante, pues esta teoría propugna que el líder debe combinar adecuadamente sus habilidades, talentos, recursos, emociones y energía propia y de sus colaboradores, para propiciar logros previstos. Es definido por Robbins (2004) como: "la capacidad de crear y articular una visión realista, atractiva y creíble del futuro de la organización o la unidad organizacional que cree y mejora a partir del presente" (p. 344). El líder visionario debe poseer mínimo tres (3) cualidades: explicar su visión a las personas, tener un comportamiento cónsono con la visión propuesta y extender esta visión más allá del ámbito donde ejerce su función y liderazgo.

Para complementar, Valdés (2002), refiere la importancia del líder para estimular el desarrollo de la riqueza intelectual. A tal efecto menciona como características resaltantes (no limitantes) del líder del futuro, las siguientes: su capacidad para estimular el desarrollo intelectual de los seguidores; incorporación de nuevas experiencias y conocimientos al ámbito donde se desenvuelve; adecuado uso de su carisma y presencia física; responder a las nuevas exigencias con flexibilidad y rapidez; asumir responsabilidades, obtener y compartir información, mantener adecuadas relaciones sociales y de comunicación; el autor antes mencionado sostiene lo siguiente:

Pasa el tiempo, se hace historia, la humanidad avanza, las instituciones evolucionan, pero a su vez más el éxito o fracaso dependerán de la calidad de liderazgo ejercido por los hombres que encuentran una mejor manera de hacer las cosas y en su capacidad para influir sobre sus seguidores y sobre su entorno (p. 496).

Dicha opinión, afirma la necesidad de que el líder tenga una visión del futuro para evolucionar y adaptarse a los continuos cambios del entorno y en todo caso ejercer más la influencia que el poder, guiar más que mandar, reafirmar el sentido de pertenencia a la organización de sus colaboradores sin obviar su individualidad e independencia, así como propiciar constantemente el desarrollo profesional y personal propio y de sus seguidores. En resumen, la postura de este autor tiende hacia la integración entre lo descrito como líder transaccional, visionario y transformador.

Como resultante, de lo expuesto en este apartado dan forma a la figura del líder y su evolución. De modo que, ayuda a la comprensión de los diferentes perfiles y así, cuando se procedió al análisis de la visión de los seguidores, fue más fácil determinar las características según las percepciones obtenidas. De lo antes tratado también puede extraerse de manera reflexiva que, las competencias de liderazgo organizacional se deben convertir en el eje central a través del cual los que dirigen las organizaciones, logren en sus seguidores, motivación, compromiso, disciplina y por lo tanto conductas que los lleven a cumplir con lo propuesto organizacionalmente. 


\section{2.- Describir el proceso de comunicación entre el líder y seguidores dentro de la organización}

La comunicación es definida por los seguidores como: buena, fluida, abierta, directa, que ocurre en los diferentes niveles y formas. En este sentido, dijeron que se desarrolla bajo principios, es inductiva, cordial y respetuosa, debido a que, en esta, se aplican los valores y principios de respeto mutuo entre el liderazgo y sus seguidores. También, se manifestó que en los niveles descendentes de liderazgo algunas veces se torna evasiva.

Para comprender este proceso entre el liderazgo y los seguidores, Mc Entee Eileelen citado por Solórzano (2010) expresa lo siguiente: "El hombre no habla simplemente para expresar emociones o puntos de vista, sino para estimular una respuesta en los demás, y para influir en sus actitudes y acciones" (p.38). Es innegable que, para entender el proceso de comunicación entre las personas que se relacionan dentro de una organización hay que ir más allá de la definición tradicional, pues se corre el riesgo de ser demasiado superficial y primario. Hoy por hoy, la comunicación se utiliza como un elemento para resolver problemas y desarrollar nuevas perspectivas difusionistas y formativas dentro de la estructura organizacional.

De modo que, más allá de un esquema, se hace necesario la interpretación de los hechos y las acciones derivadas de la misma, así se conduce a la riqueza en el proceso comunicacional. Sin olvidar que, donde interactúe el ser humano, su comunicación debe ser interpretada bajo los referentes de la formación organizacional, cultura y contexto inmediato, sobre todo si es inestable o cambiante.

Como consecuencia, ocurre el proceso de formación social como un asunto histórico, en el cual se analizan y entienden la manera en que se consolidan las tradiciones, formas de valores y afrontamiento de la realidad, así como, las formas de relacionarse, es decir, la cultura general del sector social llamado Venezuela. Por lo tanto, se hace posible analizar y comprender la manera en que se consolidan las tradiciones, valores y el modo de coexistir con la realidad, intrínsecamente en las formas de relacionarse. Dentro de este campo, Andrade (2005), señala en su aporte acerca de los tipos de comunicación interna cuatro dimensiones:

Horizontal, la cual ocurre entre el liderazgo del mismo nivel, la Ascendente y Descendente que se dan entre líderes y seguidores de manera continua y la Cruzada, que ocurre entre las diferentes unidades de la organización en lo lineal o diferentes niveles (p. 56)

Es demostrable, una interrelación que modera los procesos comunicacionales entre en liderazgo y sus seguidores. Lo que es reforzado por Fernández en lo expuesto anteriormente acerca del flujo de la comunicación confirma que, esto permite agilizar el flujo de los mensajes entre ambas figuras, influenciar en las opiniones, actitudes y conductas de los seguidores a fin de alcanzar los objetivos. 


\section{3.- Generar una síntesis interpretativa de la importancia que tiene la percepción de los seguidores acerca del liderazgo; en la búsqueda de comprender de manera acertada las interacciones de estas dos figuras en el contexto venezolano.}

Bajo la premisa de que el grupo de Empresas Polar, C. A. una empresa emblemática y tradicional para los venezolanos, es relevante comprender que, de cierto modo, se encuentra afectada por los conflictos de un contexto interno tan inestable y cambiante en los aspectos sociales-políticoseconómicos, que han penetrado en la interacción del contexto interno. A razón de que todas las organizaciones están compuestas de seres humanos sobre los cuales influye el entorno.

Sin embargo, la organización busca mantener su imagen y productividad, por medio de su liderazgo y seguidores, que poseen un alto sentido de pertenencia e identidad cultural. Todo ello, se convierte en una fortaleza en medio de la incertidumbre, ya que, ellos están conscientes de los riesgos, el caos económico y la hiperinflación a que se ven sometidos. Más, su liderazgo los mantiene motivados por medio de un buen proceso de comunicación, por encima de todos los pronósticos negativos del panorama del país, inclusive a nivel internacional.

Esta investigación buscó mostrar una comprensión de un fenómeno social y organizacional que ocurre en el contexto venezolano, como un modo de ofrecer un punto de partida en medio de una crisis. Es innegable lo que ocurre en Venezuela, pues, las nuevas tendencias de un liderazgo propio que se adapta al contexto y momento histórico que vive el país; estimulando también a sus seguidores a proyectar cada día más sus conocimientos, habilidades y experiencias, puede ser asumido estrategia que maximice el desempeño de sus funciones. De ese modo, éstos últimos se sentirán más valorados, motivados y cumplirán los fines propuestos.

En ese mismo orden de ideas, se hace necesario que los líderes observen de manera constante en momentos y situaciones a sus seguidores para detectar rasgos de liderazgo asumidos por estos. Siendo algo muy fundamental para el desenvolvimiento y enriquecimiento de los equipos de trabajo. Siguiendo con el tema, lo adecuado sería que los líderes se conviertan en entrenadores de sus seguidores y así, hacer posible que sus trabajadores alcancen un desempeño óptimo y se sientan orgullosos de ello. Lo cual llevaría a aumentar su influencia sobre éstos, establecer un mejor ambiente de trabajo y finalmente generar el apoyo tan necesario en las organizaciones.

En cuanto a la comunicación, aunque se considera buena, para incrementarla, se requiere el desarrollo de un lenguaje directo, efectivo y libre de prejuicios que facilite el entendimiento entre los miembros de los niveles intermedios y los más descendentes en la estructura organizacional. Así, la figura del liderazgo sería fortalecida y desmitificada de viejos modelos.

Y finalmente, las figuras tanto del liderazgo como los seguidores deben seguir evolucionando en la capacidad de adaptabilidad, para poder resolver los problemas relacionados con las exigencias cambiantes del medio interno y externo. Es decir, responder de un modo flexible y asertivo, anticipándose a las dificultades por medio de la combinación de la proactividad y reactividad, acciones 
estratégicas y adaptativas. Lo que los lleve a enfrentar lo cotidiano, encontrando sentido en medio de la incertidumbre y la complejidad que tiende al caos, de modo que la organización pueda sobrevivir en tiempos de crisis.

\section{CONCLUSIONES}

$\mathrm{E}$ $\mathrm{n}$ todos los espacios, donde los seres humanos coexisten nace la necesidad de poseer líderes que guíen las actividades, que promuevan las interrelaciones entre las personas dentro de las unidades operativas que están comprendidas por seres humanos. Pero también estos personajes, deben ser capaces de crear, innovar, desarrollar a otros y ser un modelo social adecuado en la perspectiva de quienes están bajo su conducción. En este sentido, se buscó analizar la percepción que los seguidores tienen del líder por una parte y por otra comprender el papel que juega la comunicación en esta interacción dentro de una de las organizaciones de mayor credibilidad y de alto desempeño en el país, aún dentro de un contexto convulsionado en los ámbitos político, económico y social. Pero que no escapa de la inestabilidad y trastornos cotidianos que viven los venezolanos.

Considerando a la comunicación como un eje fundamental dentro de las relaciones entre líderes y seguidores, en la aplicación de los procedimientos antes mencionados, se dilucidó lo siguiente:

La organización objeto de estudio, Empresas Polar, C. A., desde la perspectiva de los seguidores, posee un liderazgo innovador, creativo, capacitado, dinámico, perseverante y proactivo. A lo anterior se le suma la capacidad multifacética que le provee de flexibilidad y adaptación que le permite hacer frente a los cambios y retos cotidianos del contexto venezolano. Lo que indica rasgos descritos en la teoría del Liderazgo Situacional II expuesto por Kent Blanchard y Paul Hersey y sostiene como postulado, la capacidad de ejercer diferentes estilos, en diferentes contextos, es decir, un líder flexible capaz de adaptarse a las circunstancias.

En ese mismo orden de ideas, también se pudo apreciar que el liderazgo de esta organización posee influencia sobre sus seguidores, ejercitan valores en sus interacciones, y éstos a su vez, los perciben como un modelo a seguir y como consecuencia modelan su conducta, así también, los hacen conscientes de sus debilidades.

De igual manera se señaló que la comunicación es un elemento potenciador de las relaciones entre los líderes y sus seguidores, estos la perciben como un proceso determinado por las siguientes características: constante, bueno, fluido, abierto y bajo principios éticos que modelan la interacción y que cuando es manejada de manera eficaz, es capaz de aliviar las tensiones, miedos y riesgos del entorno laboral y finalmente traer bienestar.

Siguiendo con el tema, los seguidores manifestaron que el proceso de comunicación interna se da bajo el manejo de las siguientes herramientas: un mismo enfoque, adecuado manejo de la información en todos los niveles, el diálogo, así como las reuniones de orientación y comunicación que ayudan en la resolución de conflictos. Sin desestimar que, en los niveles de más descendentes de la estructura jerárquica de la organización como los operarios, se indicó cierta postura evasiva de los directivos 
dentro del proceso comunicacional, lo cual debe ser considerado y corregido para que no lo perjudique.

En cuanto al flujo de la comunicación organizacional y según lo considerado anteriormente por, se comprendió que se da en (4) sentidos entre las diversas unidades departamentales, indiferentemente de las jerarquías que existen: horizontal, ascendentes, descendente y cruzada entre los diversos departamentos, así como, dotada de una variedad de herramientas que van desde las impresas, directas, tecnológicas y en grupo. Los cuales, han sido manejados por la empresa de manera oportuna con un adecuado uso de las siguientes herramientas: Flujo continuo de información, reuniones periódicas, entrevistas personales, correspondencia interna, círculos de calidad, entre otros.

Finalmente, al tratar el tema de la percepción que los seguidores tienen acerca del liderazgo de las Empresas Polar, C.A., en el contexto actual venezolano, esta fue concebida como una organización emblemática tanto para los trabajadores como para los que habitan en este país. Donde los líderes y seguidores se encuentran comprometidos a mantener la imagen de ella. De igual manera, la reconocen como parte de la cultura para los públicos interno y externo. Sin obviar que, están conscientes de la inestabilidad económica que presenta el contexto tan cambiante en lo social-político y económico. Así como, el impacto que tiene la escasez de alimentos, las políticas públicas económicas poco acertadas por parte del Estado, las medidas jurídicas que afectan la producción y distribución de los productos. Lo cual, se traduce en tensiones y miedos que de un modo u otro impactan sobre el clima organizacional.

Vale decir que, en el momento en que se inició esta investigación, las estadísticas señaladas por el Informe de Competitividad Global del Fondo Monetario internacional (2013-2014) señalaban a Venezuela ranqueada en el puesto 134 como uno de los países menos competitivos en el mundo, de igual modo lo señaló en la posición (143) inmerso en una profunda crisis macroeconómica, en proceso de deterioro por el déficit público y la inflación.

Es evidente, que Venezuela no ofrece las condiciones adecuadas para que las empresas privadas desarrollen sus actividades económicas, ya que por lo anterior se interpreta que, es uno de los países menos competitivos y se encuentra en profunda estanflación económica. Todo lo anterior, se señala con la idea de visualizar dentro del contexto país a esta organización objeto de estudio, como una empresa que ha decidido permanecer, producir e innovar. Siendo de este modo, un ejemplo no solo como unidad productiva, sino también como un pilar fundamental de la sociedad venezolana. Fomentando así el ejemplo de persistencia y compromiso social a pesar de los malos tiempos, innovación con la puesta en el mercado de nuevos productos, fomentadora de transformaciones y obteniendo la credibilidad en de un público inmerso en un panorama poco esperanzador. 


\section{REFERENCIAS}

Andrade, H. (2005). Comunicación organizacional interna: procesos, disciplina y técnica. España: Gesbiblo S.L

Barroso, M. (2011). Autoestima del venezolano. Caracas, Venezuela: Galac

Blanchard, K., y Hersey P. (2011), Liderazgo al más alto Nivel, Bogotá, Colombia

Hernández, Fernández y Baptista (2008). Metodología de la investigación. 4ta. Edición. México. Mc GrawHill/Interamericana Editores, S.A

Kreitner, y Kinicki, (2008). Comportamiento de las organizaciones. 4ta. Edición. España. Mc GrawHill/Interamericana de España, S.A.

Robbins, S. (2004). Comportamiento organizacional. México: Pearson Prentice Hall

Traducido por: Dávila, J. Thiebaut, Carlos. Conceptos fundamentales de la Filosofía, Alianza Editorial, Madrid, 1998)

Valdés, L. (2002). La re-evolución empresarial del siglo XXI. Conocimiento y capital intelectual: las nuevas ventajas competitivas de la empresa. Colombia: Grupo Editorial Norma

IESA. (2016). El gerente venezolano como líder. Consultado en junio 2016. Disponible en: http://www.iesa.edu.ve/inicio/2016-abril01/2785=el-gerente-venezolano-como-lider
López, D. (2011). Los 5 niveles del liderazgo según John Maxwell. Artículo para el blog. Pensamiento imaginativo. Consultado en enero 2016. http://manuelgross.bligoo.com/20111024-los-5niveles-del-liderazgo-segun-john-maxwell

Pardo, F. (2015). Reflexiones sobre el liderazgo: redefinición del liderazgo del siglo XX. Boletín de la Universidad Rafael Belloso Chacín Volumen No. 1. (P. 28). Consultado en junio 2016. Disponible en:

URBEhttp://www11.urbe.edu/boletines/extension/ $? \mathrm{p}=28$

Pernía, A. (2013). 100 Gerentes más exitosos del 2013. Artículo en línea de la revista Gerente, edición especial anual 2013. Disponible en: http://www.gerente.com/detarticulo.php?CodArtic I=929 Consultado en abril de 2014

Solórzano, A. (2010). Intervención para mejorar las habilidades de feedback del nivel supervisor de un centro de distribución. Caracas, Venezuela. Consultado en diciembre 2015. Disponible en: http://biblioteca2.ucab.edu.ve/anexos/biblioteca/ marc/texto/AAR8442.pdf 